\title{
The influence of periodic shear on structural relaxation and pore redistribution in binary glasses
}

\author{
Nikolai V. Priezjev ${ }^{1,2}$ and Maxim A. Makeev ${ }^{3}$ \\ ${ }^{1}$ Department of Mechanical and Materials Engineering, \\ Wright State University, Dayton, $\mathrm{OH} 45435$ \\ ${ }^{2}$ National Research University Higher School of Economics, Moscow 101000, Russia and \\ ${ }^{3}$ Department of Chemistry, University of Missouri-Columbia, Columbia, MO 65211
}

(Dated: August 29, 2018)

\begin{abstract}
The evolution of porous structure, potential energy and local density in binary glasses under oscillatory shear deformation is investigated using molecular dynamics simulations. The porous glasses were initially prepared via a rapid thermal quench from the liquid state across the glass transition and allowed to phase separate and solidify at constant volume, thus producing an extended porous network in an amorphous solid. We find that under periodic shear, the potential energy decreases over consecutive cycles due to gradual rearrangement of the glassy material, and the minimum of the potential energy after thousands of shear cycles is lower at larger strain amplitudes. Moreover, with increasing cycle number, the pore size distributions become more skewed toward larger length scales where a distinct peak is developed and the peak intensity is enhanced at larger strain amplitudes. The numerical analysis of the local density distribution functions demonstrates that cyclic loading leads to formation of higher density solid domains and homogenization of the glass phase with reduced density.
\end{abstract}

PACS numbers: 34.20.Cf, 68.35.Ct, 81.05.Kf, 83.10.Rs 


\section{INTRODUCTION}

The development of efficient strategies to synthesize hierarchically structured porous materials with superior physical and mechanical properties is important for a variety of applications including adsorption, catalysis, separation, and medical devices [1]. Recent atomistic and continuum simulation studies have demonstrated that plastic deformation of metallic glasses with a regular array of pores is facilitated by nucleation of shear bands at the pore surfaces and their subsequent propagation along the planes populated by pores [2] 8 . It was recently found that under steady shear, the random porous structure in binary glasses becomes significantly transformed and dominated by a few large pores, and the shear modulus is a strong function of porosity [9, 10]. It was later shown that tensile loading at constant volume leads to pore coalescence and breaking of the glass in the regions of largest spatial extent of reduced density [11], while a nearly homogeneous amorphous material can be formed during extended strain at constant pressure [12]. Upon compression, the pore shape is initially deformed, and, at large strain, adjacent pores coalesce with each other, thus forming homogenous solid domains that provide an enhanced resistance to deformation [13]. However, the behavior of porous glasses during more complex, time-dependent deformation protocols remains relatively unexplored.

In recent years, atomistic simulations of amorphous solids under cyclic shear were especially useful in elucidating molecular mechanisms of the yielding transition, strain localization and mechanical annealing [14 30]. Here we highlight a few key results. Particularly, it was shown that below yield, binary glasses prepared with relatively large quenching rates can be mechanically annealed toward lower potential energy states, and the energy levels that can be accessed after hundreds of shear cycles become deeper at larger strain amplitudes [15, 22, 27 30]. In turn, the yielding transition typically occurs after a number of transient cycles, resulting in a formation of a shear band across the system, and, in addition, the critical strain amplitude decreases with increasing temperature [22, 23, 28, 29]. It was also found that above the yielding point, periodic shear causes irreversible deformation, plastic flow, and enhanced particle diffusion, while the system is relocated to higher potential energy states [14-26, 28, 29]. However, the effect of periodic deformation on structural and mechanical properties of phase-separated systems including porous glasses and gels remains to be fully understood. 
In this paper, we perform molecular dynamics simulations to study porous glasses subjected to oscillatory shear deformation. The porous glasses are formed during a coarsening process at low temperature after a rapid thermal quench at constant volume. It will be shown that in the absence of deformation, the potential energy gradually decreases over time, the glass phase becomes denser, and the pore size distributions are slightly skewed toward larger length scales. With increasing strain amplitude, the evolution of the porous structure after thousands of shear cycles becomes more apparent, resulting in the formation of a pronouncing peak in the distribution of larger pore sizes.

The rest of the paper is organized as follows. The preparation procedure, deformation protocol, and parameter values are presented in the next section. The analysis of the porous structure and pore size distributions as well as potential energy series and shear stress are described in section III. The results are briefly summarized in the last section.

\section{MD SIMULATIONS}

The molecular dynamics simulations described in this section were performed to study the dynamic response of a model glass to periodic shear. We consider a binary mixture model (80:20) with strongly non-additive interactions between different atom types that was originally introduced by Kob and Andersen [31]. In this model, the interaction between two atoms of types $\alpha, \beta=A, B$ is specified via the Lennard-Jones (LJ) potential as follows:

$$
V_{\alpha \beta}(r)=4 \varepsilon_{\alpha \beta}\left[\left(\frac{\sigma_{\alpha \beta}}{r}\right)^{12}-\left(\frac{\sigma_{\alpha \beta}}{r}\right)^{6}\right]
$$

where $\varepsilon_{A A}=1.0, \varepsilon_{A B}=1.5, \varepsilon_{B B}=0.5, \sigma_{A B}=0.8$, and $\sigma_{B B}=0.88$, and $m_{A}=m_{B}$ [31].

The cutoff radius is set to $r_{c, \alpha \beta}=2.5 \sigma_{\alpha \beta}$ to increase computational speed. The following convention is adopted for the reduced units of length, mass, energy, and time: $\sigma=\sigma_{A A}$, $m=m_{A}, \varepsilon=\varepsilon_{A A}$, and $\tau=\sigma \sqrt{m / \varepsilon}$, respectively. The total number of atoms is $N=300000$. The equations of motion were solved numerically using the velocity-Verlet scheme [32] with the integration time step $\triangle t_{M D}=0.005 \tau$ [33].

We next briefly describe the preparation protocol for the porous glass at constant volume, which is identical to the simulation procedure used in the recent MD studies [9, 11- 13, 3436]. The binary mixture was first thoroughly equilibrated at constant volume and at the temperature of $1.5 \varepsilon / k_{B}$ that is larger than the glass transition temperature $T_{g} \approx 0.435 \varepsilon / k_{B}$. 
Here, $k_{B}$ denotes the Boltzmann constant. In turn, the porous glass was formed during the coarsening process at constant volume and temperature $T_{L J}=0.05 \varepsilon / k_{B}$ after an instantaneous thermal quench from the liquid state. As a result, after the time interval of $10^{4} \tau$, the porous structure is developed inside the amorphous material [9, 11, 13, 34, 36]. In the present study, due to limited computational resources, we only considered one realization of disorder for a sample with the average glass density $\rho \sigma^{3}=0.5$.

Following the preparation protocol, the porous glass was subjected to oscillatory shear deformation at constant volume. More specifically, the time-periodic strain was applied using the following relation:

$$
\gamma(t)=\gamma_{0} \sin (2 \pi t / T),
$$

where $\gamma_{0}$ is the strain amplitude and $T$ is the oscillation period. To avoid confusion, in what follows, the temperature is denoted by $T_{L J}$, whereas the oscillation period is referred to as $T$. We considered the values of strain amplitude from $\gamma_{0}=0$ to 0.012 and the oscillation period $T=500 \tau$. The simulations were carried out during 2000 shear cycles for each value of $\gamma_{0}$. Note that the value $\gamma_{0}=0$ corresponds to a quiescent sample that was aged at constant volume during the time interval of $2000 \mathrm{~T}=10^{6} \tau$. The temperature $T_{L J}=0.05 \varepsilon / k_{B}$ was regulated using the Nosé-Hoover thermostat 33. In addition, the Lees-Edwards periodic boundary conditions were applied in the plane of shear, while the usual periodic boundary conditions were imposed along the vorticity direction [33]. We comment that considerable computational resources (hundreds of processors) were required to simulate the system of 300000 particles for $2 \times 10^{8}$ MD steps. A relatively large system size was chosen to avoid finite size effects [34, 35]. During production runs, the potential energy, shear stress, pressure and temperature as well as atomic configurations after each cycle were saved for the postprocessing analysis.

\section{RESULTS}

We begin the discussion by briefly reviewing the process of formation of the porous glass and its properties after the initial stage of phase separation. It was previously shown that after an instantaneous isochoric quench to a low temperature well below $T_{g}$, the glass-

forming liquid undergoes simultaneous phase separation and solidification of the material [34, 35]. Moreover, the phase separation kinetics at sufficiently low temperatures crosses over 
after about $10^{2} \tau$ from a power-law increase to a logarithmically slow domain growth [34, 35]. The resulting porous structure after the time interval of $10^{4} \tau$ at $T_{L J}=0.05 \varepsilon / k_{B}$ for the sample with the average glass density $\rho \sigma^{3}=0.5$ is typically characterized by a percolating solid carcase and a complex porous network [36]. It should be emphasized that the conditions of constant volume and low temperatures generally result in the formation of the porous structures at negative pressures [34 36]. Remarkably, it was found that the ratio of the pressure and temperature scales as $P / T_{L J} \sim \rho^{2.5}$ in a wide parameter range [36]. In particular, the average value of the pressure for the glass density $\rho \sigma^{3}=0.5$ at $T_{L J}=$ $0.05 \varepsilon / k_{B}$ is $P \approx-0.15 \varepsilon / \sigma^{3}$, and the system is effectively under tension in a confined geometry 36].

The variation of the potential energy per atom as a function of time, expressed in periods, is shown in Fig. 1 for different strain amplitudes. When plotted on the log scale, it becomes evident that in each case the potential energy gradually decreases over consecutive cycles, and the steady states of deformation at the lowest energy levels are yet to be reached. Due to limited computational resources, we examine the dynamic response to periodic shear in porous glasses only during the first 2000 cycles. Thus, the case $\gamma_{0}=0$ represents an aging process at constant volume in the undeformed porous glass, which is essentially a continuation of the coarsening dynamics extended to a time interval of $2000 T=10^{6} \tau$. As will be shown below, even in the absence of mechanical agitation, the porous structure and the glass phase undergo a noticeable transformation during $10^{6} \tau$. We comment that the relative decrease in the potential energy at constant volume in the quiescent sample (shown in Fig. 1) is significantly smaller than the energy decrease during aging at constant pressure, which occurs due to volume decrease and densification of the glass phase in the latter case [12]. Furthermore, with increasing strain amplitude, the porous systems relocate to lower levels of the potential energy, indicating structural changes in the glass phase and pore redistribution (discussed below). The effect of cyclic loading on the lowest energy levels for different strain amplitudes is summarized in the inset to Fig.1.

Next, the shear stress during 2000 cycles is shown in Fig.2 for selected values of the strain amplitude. Note that the data are shifted vertically for clarity. It can be observed that after about a hundred transient cycles, the amplitude of stress oscillations only weakly depends on the cycle number. Somewhat surprisingly, the stress amplitude after about 400 
cycles slightly increases with time for $\gamma_{0}=0.10$, while it steadily decreases for $\gamma_{0}=0.08$. It can also be seen that with increasing $\gamma_{0}$, the amplitude of stress oscillations increases up to $\gamma_{0}=0.04$, indicating yielding and plastic flow during oscillations with the strain amplitude $\gamma_{0}=0.06$ and above. We comment that determination of the exact yielding point and identification of the strain localization is not the main focus of the study, as we examine long-term structural changes in the glass phase and porous network. Finally, a more detailed view of the stress-strain response during the first 100 cycles is presented in Fig. 3. The local maximum in stress amplitude during the first few cycles for $\gamma_{0} \geqslant 0.07$ is reminiscent to the behavior observed for poorly annealed, homogeneous glasses cycled with the strain amplitude $\gamma_{0}=0.06$, when the system was deformed nearly reversibly with a relatively large stress amplitude for about a hundred cycles before yielding and formation of a shear band [28]. As expected, the typical amplitude of stress oscillations for the porous glass at a given $\gamma_{0}$ is significantly lower than the stress amplitude for homogeneous binary glasses with the average density $\rho \sigma^{3}=1.2$ reported in the previous studies [23, 27, 28].

The representative snapshots of the porous glasses cycled with different strain amplitudes are displayed in Figs. 4.7. The atomic configurations are presented after the first, tenth, 100th and the last cycle at zero strain. It can be seen that the porous structure in the quiescent glass, shown in Fig.4, is composed of small isolated pores and extended, highly tortuous channels. During the aging process at constant volume, the shape of the pores is only slightly changed even after the longest time interval $t=2000 T$, as shown in Fig.4. It can be further observed that with increasing strain amplitude, the evolution of the porous network becomes more apparent as larger pores are formed. Moreover, at the largest strain amplitude $\gamma_{0}=0.12$, the small-size pores become essentially expelled from the glass phase and solid strands are formed across the system due to periodic boundary conditions and the constraint of constant volume (see Fig.7). These structural changes in periodically driven porous glasses can be quantified by computing the distribution of spheres with different diameters that fill the porous network.

The evaluation of the pore size distribution functions was performed using the opensource Zeo++ software developed at the Lawrence Berkeley National Laboratory [37 [39]. More specifically, the total volume of the system was decomposed into Voronoi cells utilizing the $\mathrm{VORO}++$ software library [40]. As a results, the space around each atom is surrounded 
by a polyhedral cell with edges that are equidistant from neighboring atoms in a periodic simulation domain. Among other structural characteristics, the probe accessible regions of the void-space network can be identified using a modification of the Dijkstra shortest path algorithm [41]. In the present study, the number of samples per atom is 50000 and the probe radius is set to $0.3 \sigma$.

The pore size distribution functions are presented in Fig. 8 for strain amplitudes $\gamma_{0}=0$, 0.04, 0.06 and in Fig.9 for $\gamma_{0}=0.07,0.08$, and 0.12. In each case, the data were collected after the indicated number of cycles at zero strain. Note that the black curve denotes the pore size distributions in the porous glass right after the thermal quench $(t=0)$ and it is the same in all panels in Figs.8 and 9. It can be seen from Fig.8(a) that in the quiescent glass, the distribution functions remain nearly the same during the time interval $t=10 T$, while larger pores are gradually developed after the waiting time $t=2000 \mathrm{~T}$. The effect is rather subtle and it can hardly be detected by visual examination of the atomic configurations shown in Fig.4. By contrast, in deformed glasses, the change in PSD functions becomes apparent even after the first shear cycle, and the difference between curves at $t=0$ and $t=T$ is amplified with increasing strain amplitude. Moreover, a pronounced peak at increasingly large length scales is developed at larger strain amplitudes, and, in addition, the peak height becomes larger with increasing either cycle number or strain amplitude. The largest transformation of the porous structure can be observed for the strain amplitude $\gamma_{0}=0.12$ shown in Fig.7, where a large void space is formed around a compact glass phase after 2000 cycles. The appearance of such system-spanning void is reflected in the highest peak at $d_{p} \approx 38 \sigma$ shown in Fig.9(c).

To reveal a more complete picture of a porous material response to periodic loading, in what follows, we focus on the analysis of the local glass density. Specifically, we compute the local density distribution functions in the solid-state domains. The local density, $\langle\rho\rangle_{R}$, is defined as the number of atoms located within the given radial range centered on a site of the cubic lattice $L \in R^{3}$. An analytical expression for the quantity can readily be built using the following procedure. First, for each lattice site, we define a closed ball, $B_{R}=\left\{R \in \mathbb{R}^{3}, \sum_{j=1}^{3} R_{j}^{2} \leqslant R_{0}^{2}\right\}$, where $R_{0}=\left|\vec{R}_{0}\right|$ is a fixed non-zero rational number. Then, the on-site local density for an atomic ensemble consisting of $N$ atoms can readily be obtained as $\langle\rho\rangle_{R}=1 / B_{R} \int d R^{3} \delta\left(\vec{r}_{i}-\vec{R}\right)$, where the integral is taken over $B_{R}$ and $i=1,2, \ldots, N$ is the 
atomic index. Note that $\langle\rho\rangle_{R}$ can be regarded as a measure of deviation of the local density from the average density of a homogeneous dense glass. Following the previous analysis of porous glasses in a wide range of average glass densities [36], we used a fixed value $R_{0}=2.5 \sigma$ in the present study.

In Fig. 10, we plot the local density distribution functions, computed for porous glasses loaded with strain amplitudes in the range $0 \leqslant \gamma_{0} \leqslant 0.12$. As can be deduced from Fig. 10 , the periodic loading causes substantial changes in the structural properties of the solid domains. The effects differ for smaller density domains $\left(\rho \sigma^{3}<0.5\right)$ and denser domains, in particular, those where $\rho \sigma^{3}$ is close to the corresponding density of a homogeneous glass. These structural changes also depend strongly on the strain amplitude and cycle number. In particular, a significant homogenization of the solid domains occurs in the solid regions with smaller density. On average, these regions become less abundant in the driven systems. Correspondingly, the intensity of the peak in the distribution functions, located near the

average density of dense non-porous glassy phase, increases (see regions $\rho \sigma^{3}>1.0$ in Fig. 10. The effect is most pronounced for the largest strain amplitude, $\gamma_{0}=0.12$, in which case the increase in the peak value can be as large as in excess of $150 \%$. By contrast, in the lower density regions, a flattening of the density distribution functions and a decrease in their magnitudes is observed. As clear from Fig. 10, the degree of flattening increases with strain amplitude in the whole region of strain amplitude variations. Overall, we find that periodic loading leads to significant homogenization of porous glasses, wherein a growth of solid domains with densities characteristic for dense non-porous glasses is observed. Further studies should focus on the effects of periodic loading on the smaller-scale defects in glasses. A work in this direction is currently in progress.

\section{CONCLUSIONS}

In summary, we performed a molecular dynamics simulation study of porous glasses subjected to cyclic mechanical loading with varied strain amplitude. The main emphasis of the study was put on temporal evolution of the void spaces and solid domains in response to periodic shear with varied amplitude and fixed period. We found that cyclic loading causes substantial rearrangements in the structures of both void spaces and solid domains. In particular, we observed that there exist a segregation of void space and solid domains, 
such that large pores are energetically favored. The effect is an increasing function of the strain amplitude. Moreover, depending on the strain amplitude, the periodic shear is found to considerably facilitate both homogenization of the solid domains in porous systems and leads to solid-domain densification, as revealed by the behavior of the local density distributions, reported on in this work. The combination of these observations allows the authors to conclude that periodic mechanical loading can potentially be employed as means for improving the structural properties of metallic glasses and/or for design of glassy materials with desired properties.

\section{Acknowledgments}

Financial support from the National Science Foundation (CNS-1531923) is gratefully acknowledged. The article was prepared within the framework of the Basic Research Program at the National Research University Higher School of Economics (HSE) and supported within the framework of a subsidy by the Russian Academic Excellence Project '5-100'. The molecular dynamics simulations were performed using the LAMMPS numerical code [33]. The distributions of pore sizes were computed using the open-source software ZEO++ [37 39$]$. Computational work in support of this research was performed at Wright State University's High Performance Computing Facility and the Ohio Supercomputer Center.

[1] X.-Y. Yang, L.-H. Chen, Y. Li, J. C. Rooke, C. Sanchez, and B.-L. Su, Hierarchically porous materials: Synthesis strategies and structure design, Chem. Soc. Rev. 46, 481 (2017).

[2] B. Sarac, B. Klusemann, T. Xiao, and S. Bargmann, Materials by design: An experimental and computational investigation on the microanatomy arrangement of porous metallic glasses, Acta Mater. 77, 411 (2014).

[3] B. Sarac, D. Sopu, E. Park, J. K. Hufenbach, S. Oswald, M. Stoica, and J. Eckert, Mechanical and structural investigation of porous bulk metallic glasses, Metals 5, 920 (2015).

[4] D. Sopu, C. Soyarslan, B. Sarac, S. Bargmann, M. Stoica, and J. Eckert, Structure-property relationships in nanoporous metallic glasses, Acta Mater. 106, 199 (2016).

[5] S. Gouripriya and P. Tandaiya, Mechanisms of compressive deformation and failure of porous 
bulk metallic glasses, Modelling Simul. Mater. Sci. Eng. 25, 045006 (2017).

[6] Y. Jiang, L. Sun, Q. Wu, and K. Qiu, Enhanced tensile ductility of metallic glass matrix composites with novel microstructure, J. Non-Cryst. Solids 459, 26 (2017).

[7] H. Y. Song, S. Li, Y. G. Zhang, Q. Deng, T. H. Xu, and Y. L. Li, Atomic simulations of plastic deformation behavior of $\mathrm{Cu}_{50} \mathrm{Zr}_{50}$ metallic glass, J. Non-Cryst. Solids 471, 312 (2017).

[8] Y. Luo, G. Yang, Y. Shao, and K. Yao, The effect of void defects on the shear band nucleation of metallic glasses, Intermetallics 94, 114 (2018).

[9] N. V. Priezjev and M. A. Makeev, Evolution of the pore size distribution in sheared binary glasses, Phys. Rev. E 96, 053004 (2017).

[10] M. Sahimi, Applications of Percolation Theory (Taylor and Francis, London, 1994).

[11] N. V. Priezjev and M. A. Makeev, Strain-induced deformation of the porous structure in binary glasses under tensile loading, Comput. Mater. Sci. 150, 134 (2018).

[12] N. V. Priezjev and M. A. Makeev, Structural relaxation of porous glasses due to internal stresses and deformation under tensile loading at constant pressure, arXiv:1808.04033 (2018).

[13] N. V. Priezjev and M. A. Makeev, Structural transformations and mechanical properties of porous glasses under compressive loading, J. Non-Cryst. Solids (2018). In press. DOI: https://doi.org/10.1016/j.jnoncrysol.2018.04.008

[14] N. V. Priezjev, Heterogeneous relaxation dynamics in amorphous materials under cyclic loading, Phys. Rev. E 87, 052302 (2013).

[15] D. Fiocco, G. Foffi, and S. Sastry, Oscillatory athermal quasistatic deformation of a model glass, Phys. Rev. E 88, 020301(R) (2013).

[16] I. Regev, T. Lookman, and C. Reichhardt, Onset of irreversibility and chaos in amorphous solids under periodic shear, Phys. Rev. E 88, 062401 (2013).

[17] N. V. Priezjev, Dynamical heterogeneity in periodically deformed polymer glasses, Phys. Rev. E 89, 012601 (2014).

[18] I. Regev, J. Weber, C. Reichhardt, K. A. Dahmen, and T. Lookman, Reversibility and criticality in amorphous solids, Nat. Commun. 6, 8805 (2015).

[19] N. V. Priezjev, Reversible plastic events during oscillatory deformation of amorphous solids, Phys. Rev. E 93, 013001 (2016).

[20] T. Kawasaki and L. Berthier, Macroscopic yielding in jammed solids is accompanied by a non-equilibrium first-order transition in particle trajectories, Phys. Rev. E 94, 022615 (2016). 
[21] N. V. Priezjev, Nonaffine rearrangements of atoms in deformed and quiescent binary glasses, Phys. Rev. E 94, 023004 (2016).

[22] P. Leishangthem, A. D. S. Parmar, and S. Sastry, The yielding transition in amorphous solids under oscillatory shear deformation, Nat. Commun. 8, 14653 (2017).

[23] N. V. Priezjev, Collective nonaffine displacements in amorphous materials during largeamplitude oscillatory shear, Phys. Rev. E 95, 023002 (2017).

[24] M. Fan, M. Wang, K. Zhang, Y. Liu, J. Schroers, M. D. Shattuck, and C. S. O'Hern, The effects of cooling rate on particle rearrangement statistics: Rapidly cooled glasses are more ductile and less reversible, Phys. Rev. E 95, 022611 (2017).

[25] S. Dagois-Bohy, E. Somfai, B. P. Tighe, and M. van Hecke, Softening and yielding of soft glassy materials, Soft Matter 13, 9036 (2017).

[26] R. Ranganathan, Y. Shi, and P. Keblinski, Commonalities in frequency-dependent viscoelastic damping in glasses in the MHz to THz regime, J. Appl. Phys. 122, 145103 (2017).

[27] N. V. Priezjev, Molecular dynamics simulations of the mechanical annealing process in metallic glasses: Effects of strain amplitude and temperature, J. Non-Cryst. Solids 479, 42 (2018).

[28] N. V. Priezjev, The yielding transition in periodically sheared binary glasses at finite temperature, Comput. Mater. Sci. 150, 162 (2018).

[29] A. D. S. Parmar, S. Kumar, and S. Sastry, Strain localisation above the yielding point in cyclically deformed glasses, arXiv:1806.02464 (2018).

[30] N. V. Priezjev, Slow relaxation dynamics in binary glasses during stress-controlled, tensioncompression cyclic loading, Comput. Mater. Sci. 153, 235 (2018).

[31] W. Kob and H. C. Andersen, Testing mode-coupling theory for a supercooled binary LennardJones mixture: The van Hove correlation function, Phys. Rev. E 51, 4626 (1995).

[32] M. P. Allen and D. J. Tildesley, Computer Simulation of Liquids (Clarendon, Oxford, 1987).

[33] S. J. Plimpton, Fast parallel algorithms for short-range molecular dynamics, J. Comp. Phys. 117, 1 (1995).

[34] V. Testard, L. Berthier, and W. Kob, Influence of the glass transition on the liquid-gas spinodal decomposition, Phys. Rev. Lett. 106, 125702 (2011).

[35] V. Testard, L. Berthier, and W. Kob, Intermittent dynamics and logarithmic domain growth during the spinodal decomposition of a glass-forming liquid, J. Chem. Phys. 140, 164502 (2014). 
[36] M. A. Makeev and N. V. Priezjev, Distributions of pore sizes and atomic densities in binary mixtures revealed by molecular dynamics simulations, Phys. Rev. E 97, 023002 (2018).

[37] R. L. Martin, B. Smit, and M. Haranczyk, Addressing challenges of identifying geometrically diverse sets of crystalline porous materials, J. Chem. Inf. Model. 52, 308 (2012).

[38] T. F. Willems, C. H. Rycroft, M. Kazi, J. C. Meza, and M. Haranczyk, Algorithms and tools for high-throughput geometry-based analysis of crystalline porous materials, Micropor. Mesopor. Mater. 149, 134 (2012).

[39] D. Ongari, P. G. Boyd, S. Barthel, M. Witman, M. Haranczyk, and B. Smit, Accurate characterization of the pore volume in microporous crystalline materials, Langmuir 33, 14529 (2017).

[40] C. H. Rycroft, VORO++: A three-dimensional Voronoi cell library in C++, Chaos 19, 041111 (2009).

[41] E. W. Dijkstra, A note on two problems in connexion with graphs, Numerische Mathematik 1, 269 (1959). 
Figures 


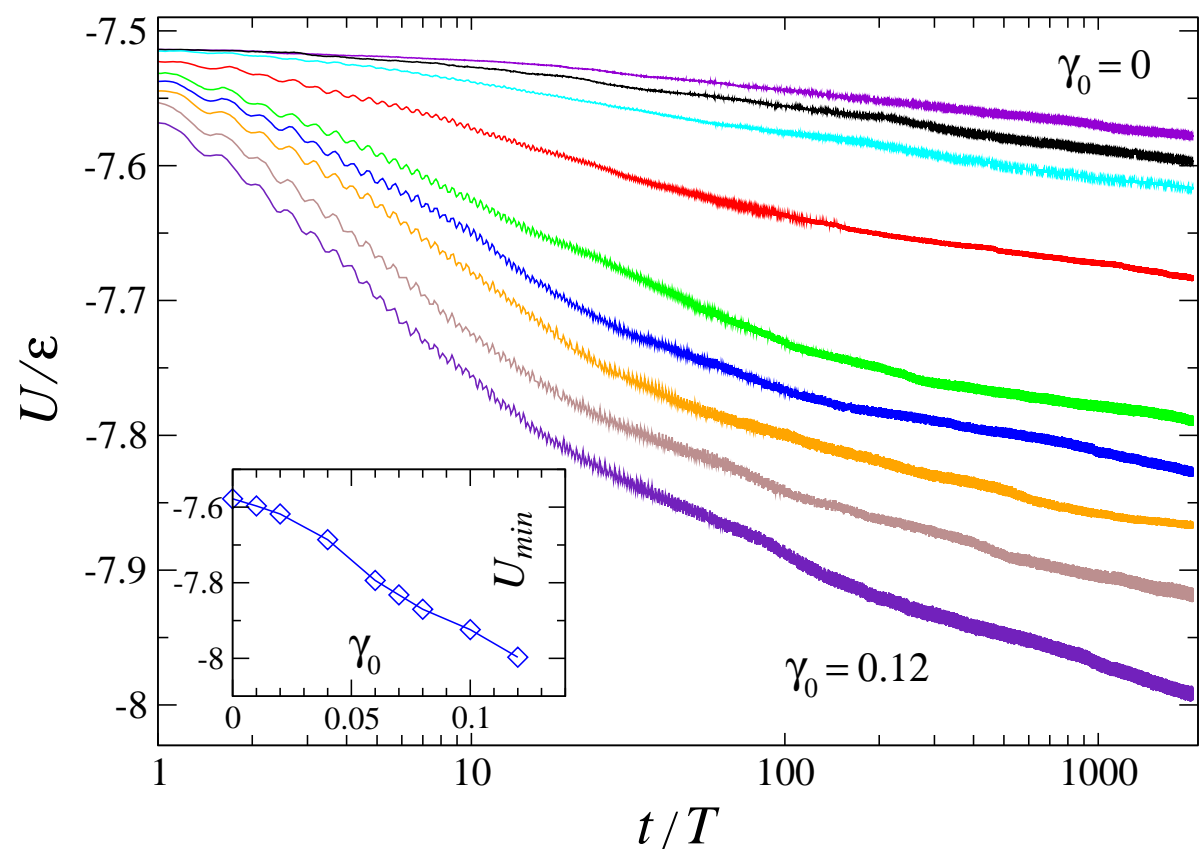

FIG. 1: (Color online) The potential energy per atom as a function of time for strain amplitudes $\gamma_{0}=0,0.01,0.02,0.04,0.06,0.07,0.08,0.10$, and 0.12 (from top to bottom). The oscillation period is $T=500 \tau$ and the average glass density is $\rho \sigma^{3}=0.5$. The inset shows the minimum of the potential energy after 2000 cycles versus the strain amplitude. 


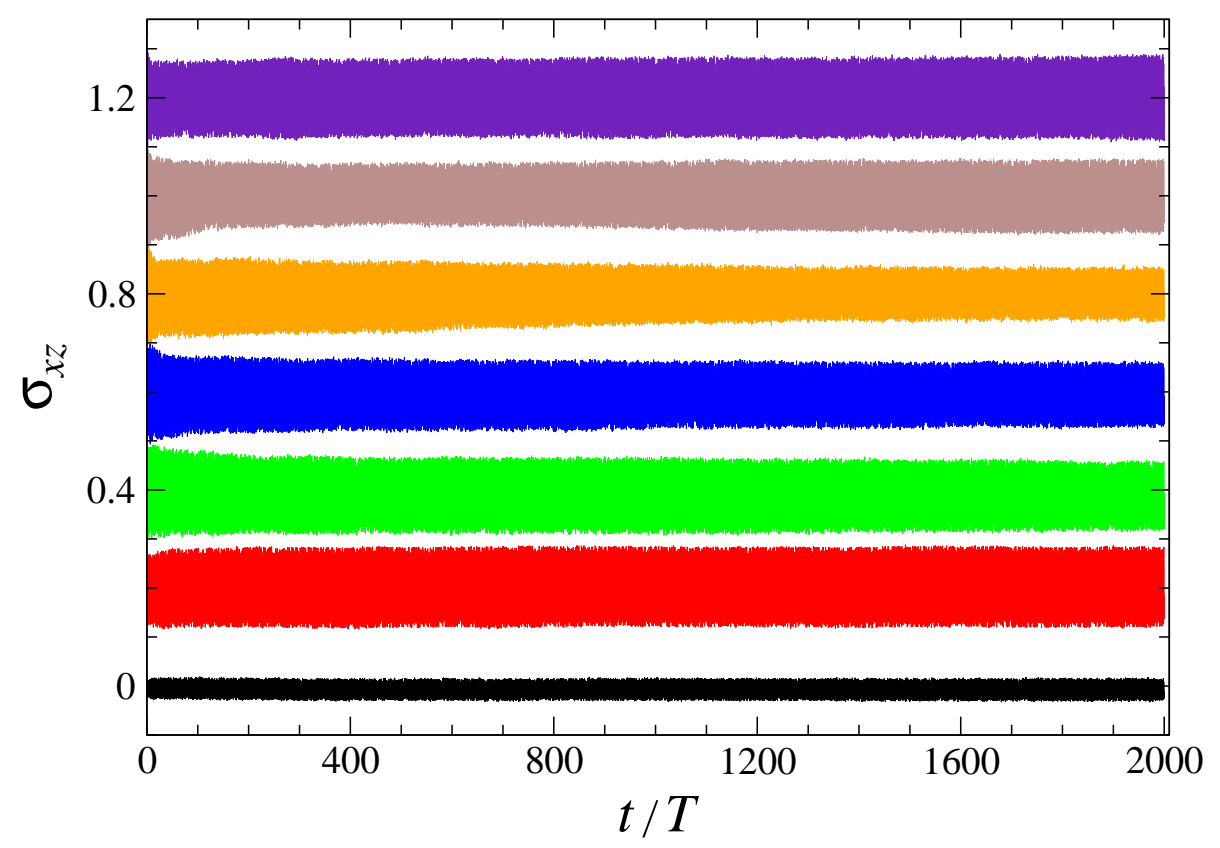

FIG. 2: (Color online) The time dependence of the shear stress $\sigma_{x z}$ (in units of $\varepsilon \sigma^{-3}$ ) for strain amplitudes $\gamma_{0}=0.01,0.04,0.06,0.07,0.08,0.10$, and 0.12 (from bottom to top). For clarity, the data are displaced upward by $0.2 \varepsilon \sigma^{-3}$ for $\gamma_{0}=0.04$, by $0.4 \varepsilon \sigma^{-3}$ for $\gamma_{0}=0.06$, by $0.6 \varepsilon \sigma^{-3}$ for $\gamma_{0}=0.07$, by $0.8 \varepsilon \sigma^{-3}$ for $\gamma_{0}=0.08$, by $1.0 \varepsilon \sigma^{-3}$ for $\gamma_{0}=0.10$, and by $1.2 \varepsilon \sigma^{-3}$ for $\gamma_{0}=0.12$. 


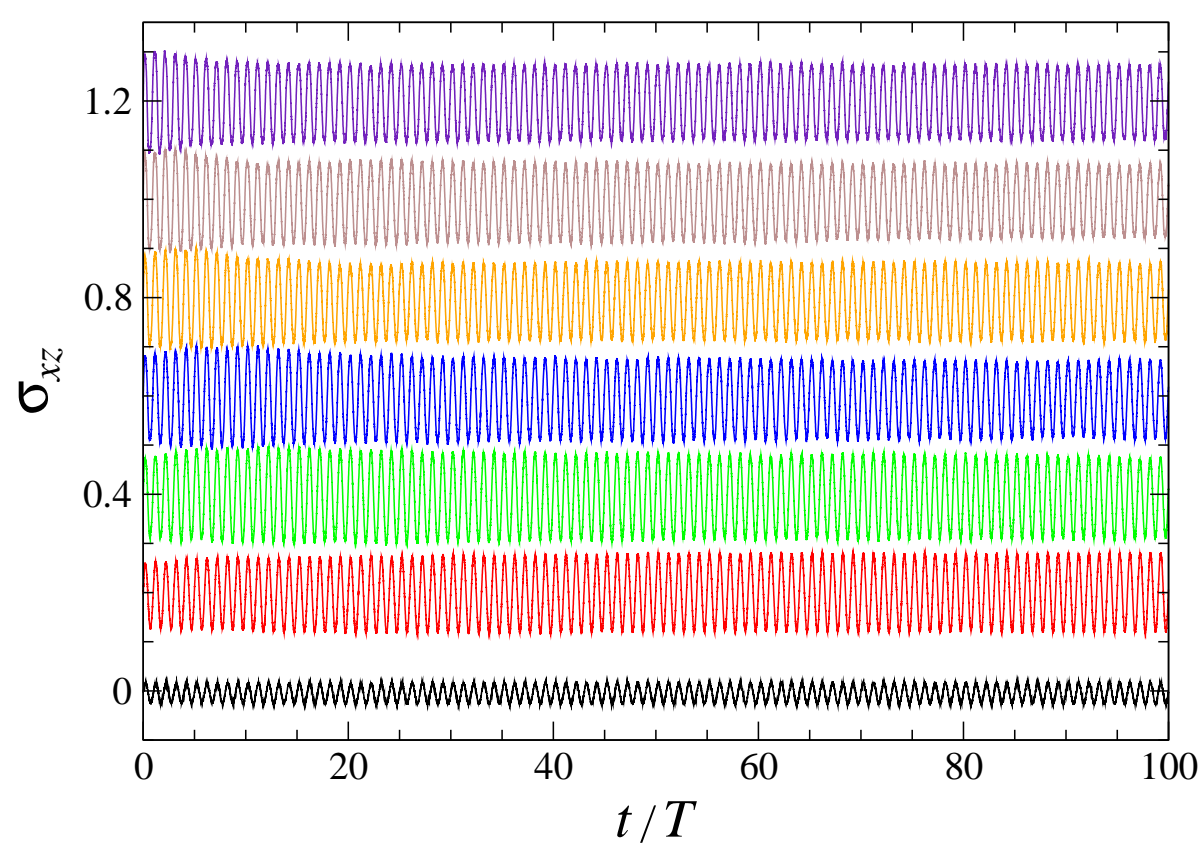

FIG. 3: (Color online) The shear stress $\sigma_{x z}$ (in units of $\varepsilon \sigma^{-3}$ ) as a function of time for strain amplitudes $\gamma_{0}=0.01,0.04,0.06,0.07,0.08,0.10$, and 0.12 (from bottom to top). The same data as in Fig. 2 but plotted for the first 100 cycles. 
(a)
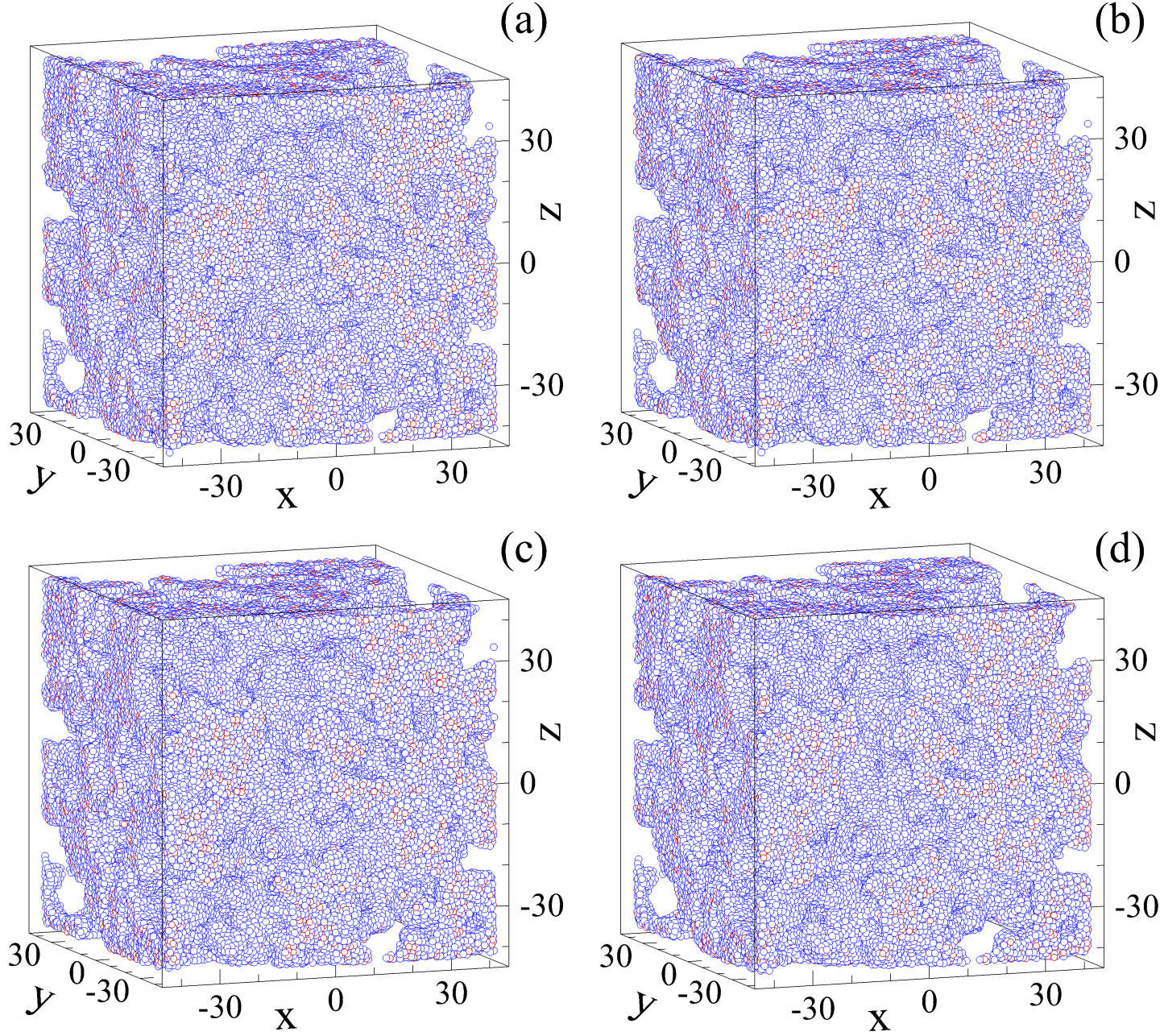

(c)

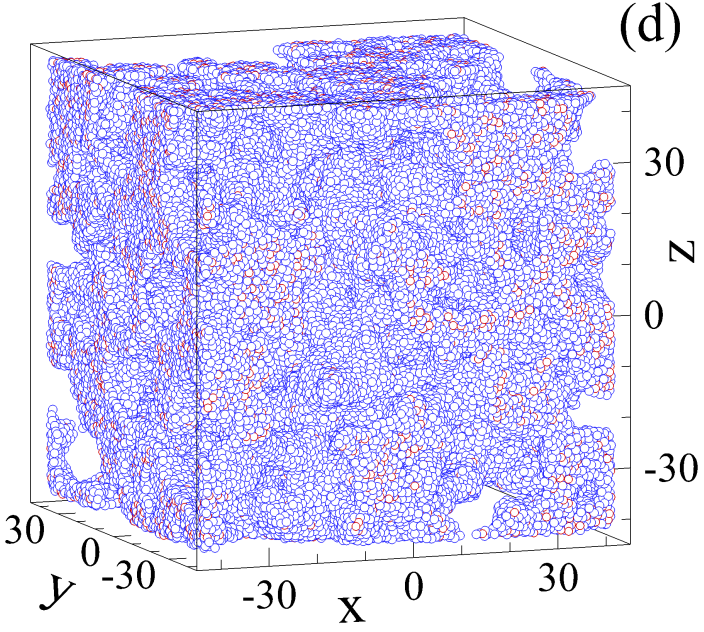

FIG. 4: (Color online) Instantaneous snapshots of the quiescent porous glass $\left(\gamma_{0}=0\right)$ after time intervals (a) $T$, (b) $10 T$, (c) $100 T$, and (d) $2000 T$. The period of oscillation is $T=500 \tau$ and the average glass density is $\rho \sigma^{3}=0.5$. 
(a)

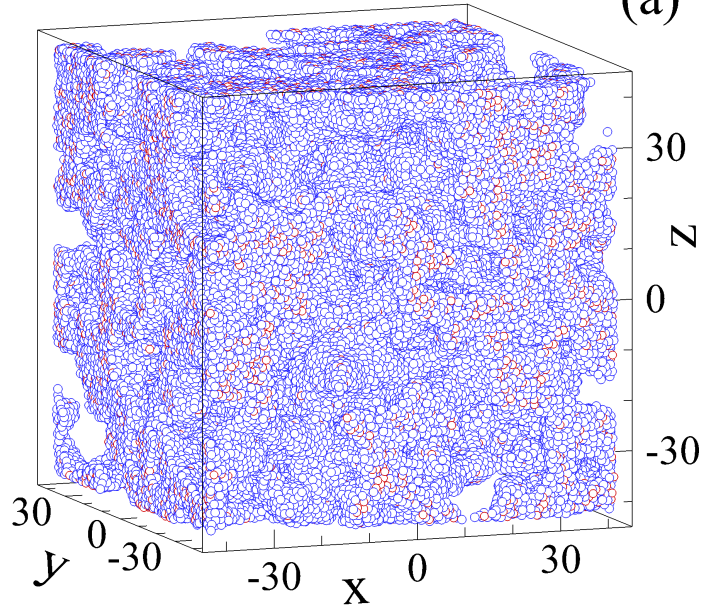

(c)

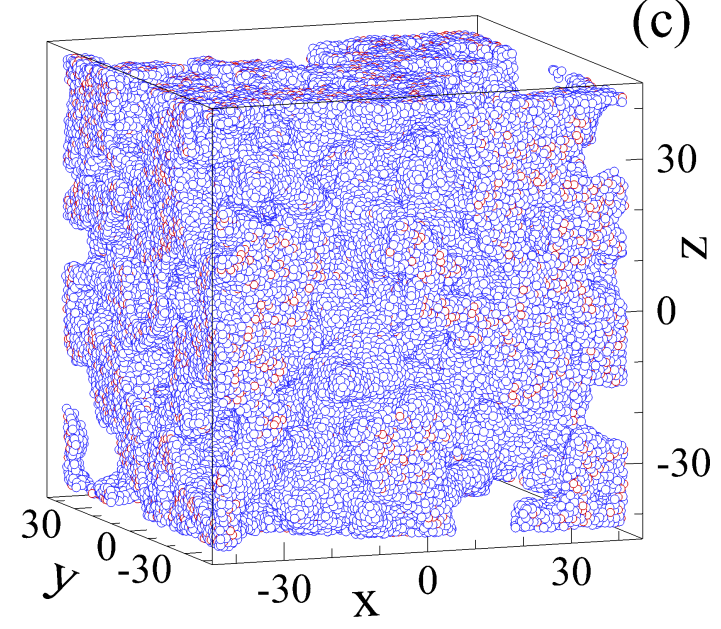

(b)

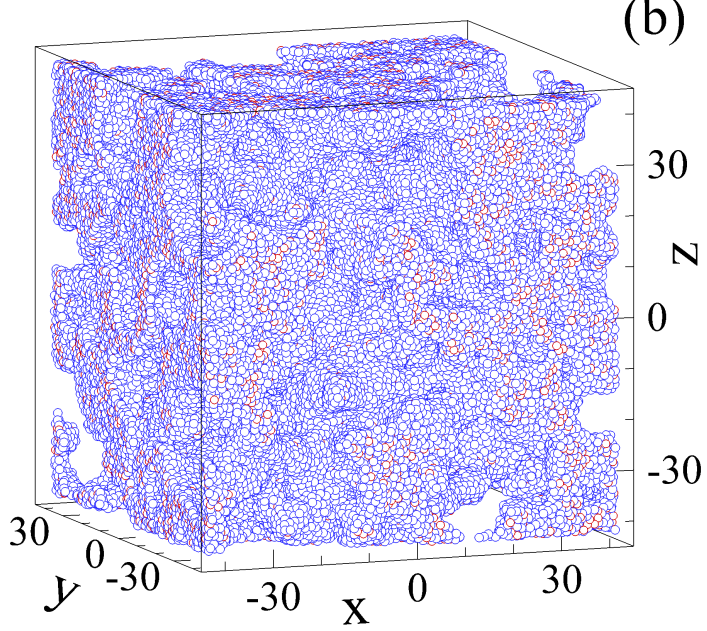

(d)

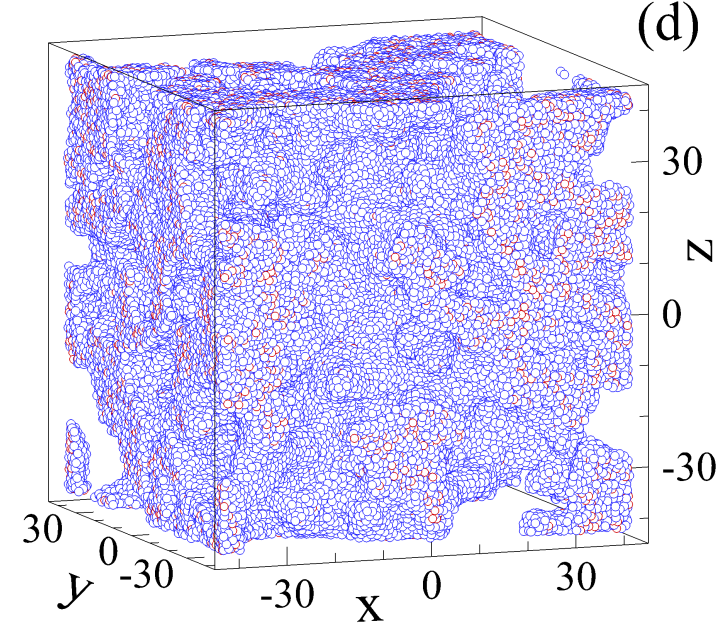

FIG. 5: (Color online) Atomic configurations of the porous glass under oscillatory shear with the strain amplitude $\gamma_{0}=0.04$ after (a) 1-st, (b) 10-th, (c) 100-th, and (d) 2000-th cycle. 
(a)
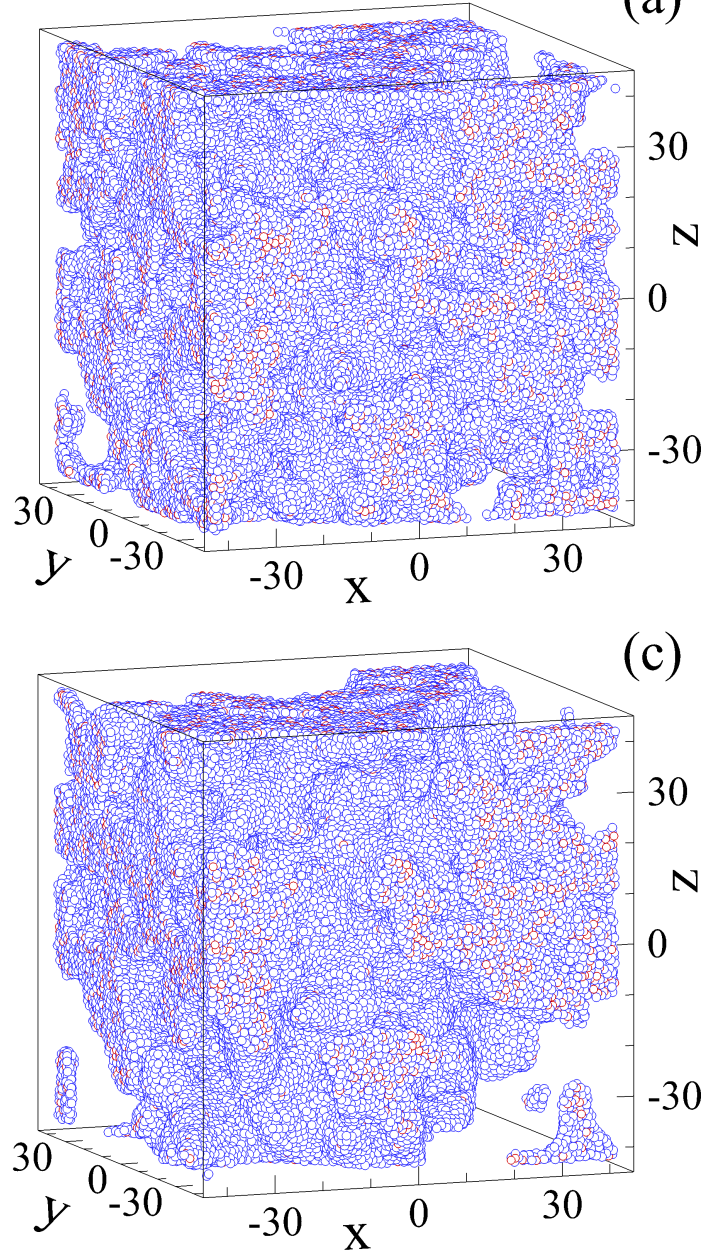

(b)

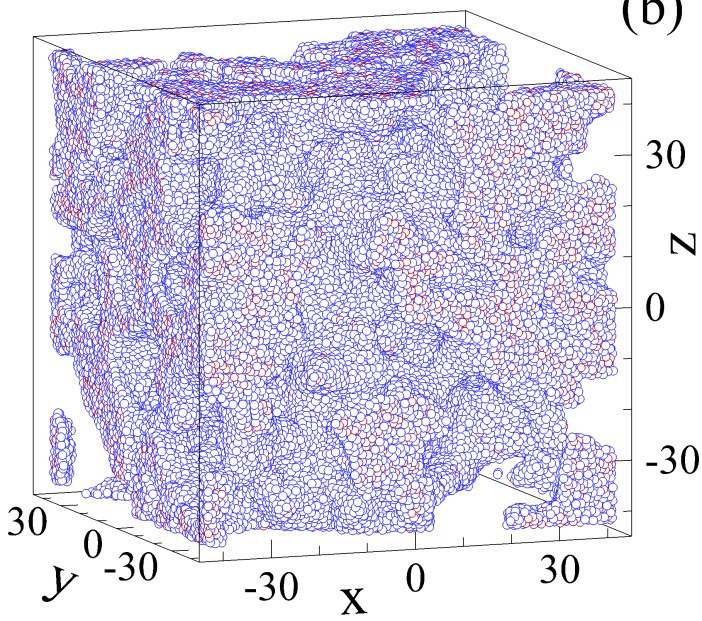

(d)

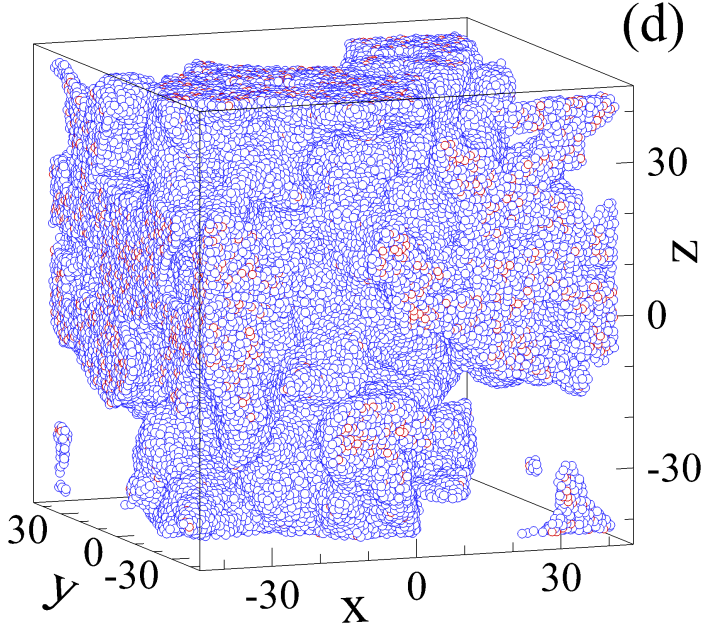

FIG. 6: (Color online) Instantaneous snapshots of the porous glass periodically deformed with the strain amplitude $\gamma_{0}=0.08$ after (a) 1-st, (b) 10-th, (c) 100-th, and (d) 2000-th cycle. 
(a)
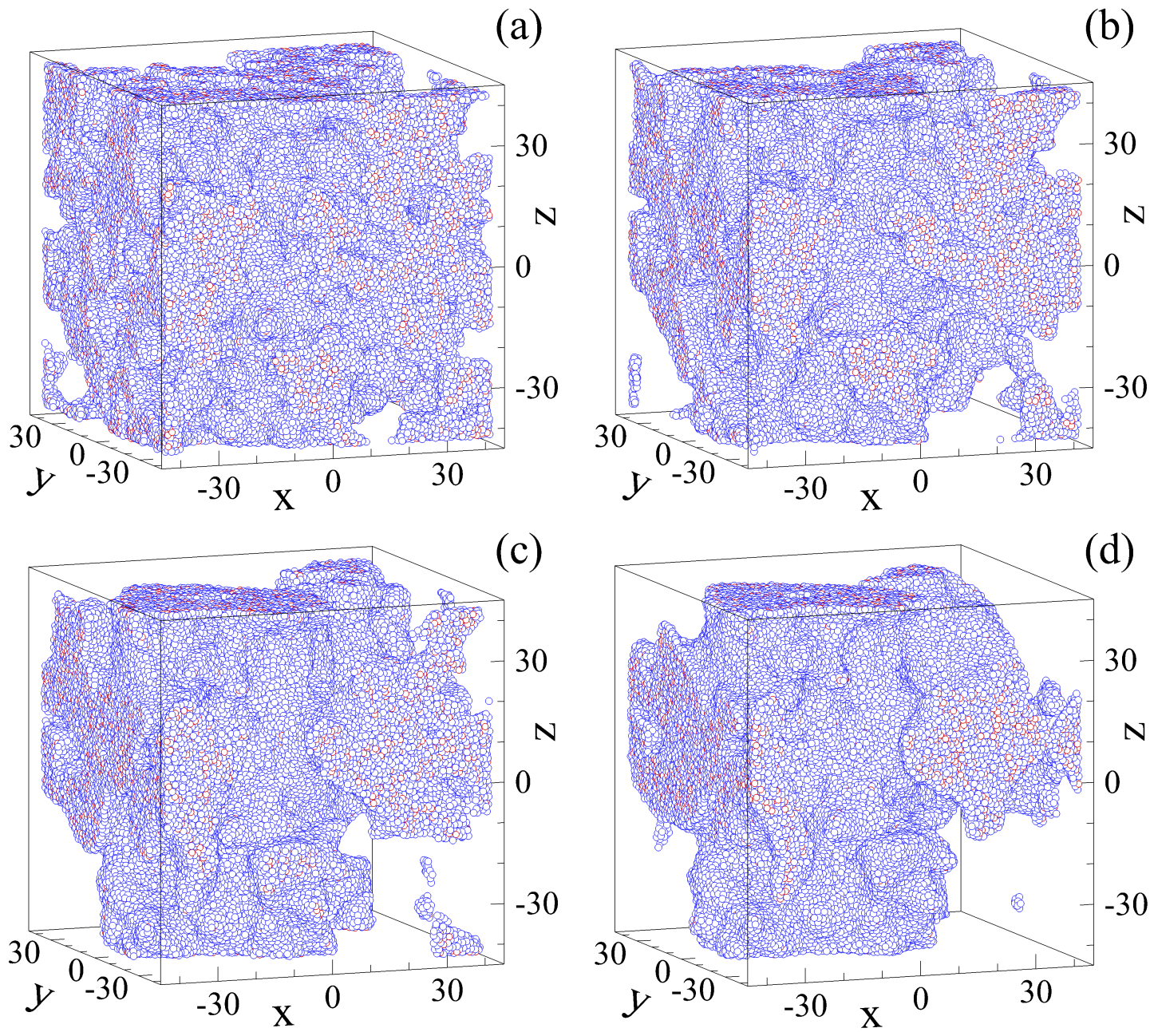

(c)

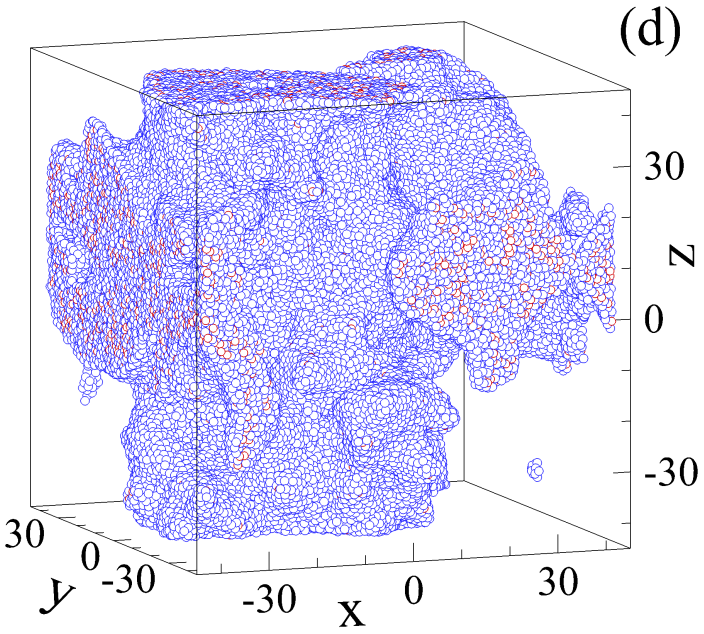

FIG. 7: (Color online) Atom positions during cyclic loading with the strain amplitude $\gamma_{0}=0.12$ after (a) 1-st, (b) 10-th, (c) 100-th, and (d) 2000-th cycle. 


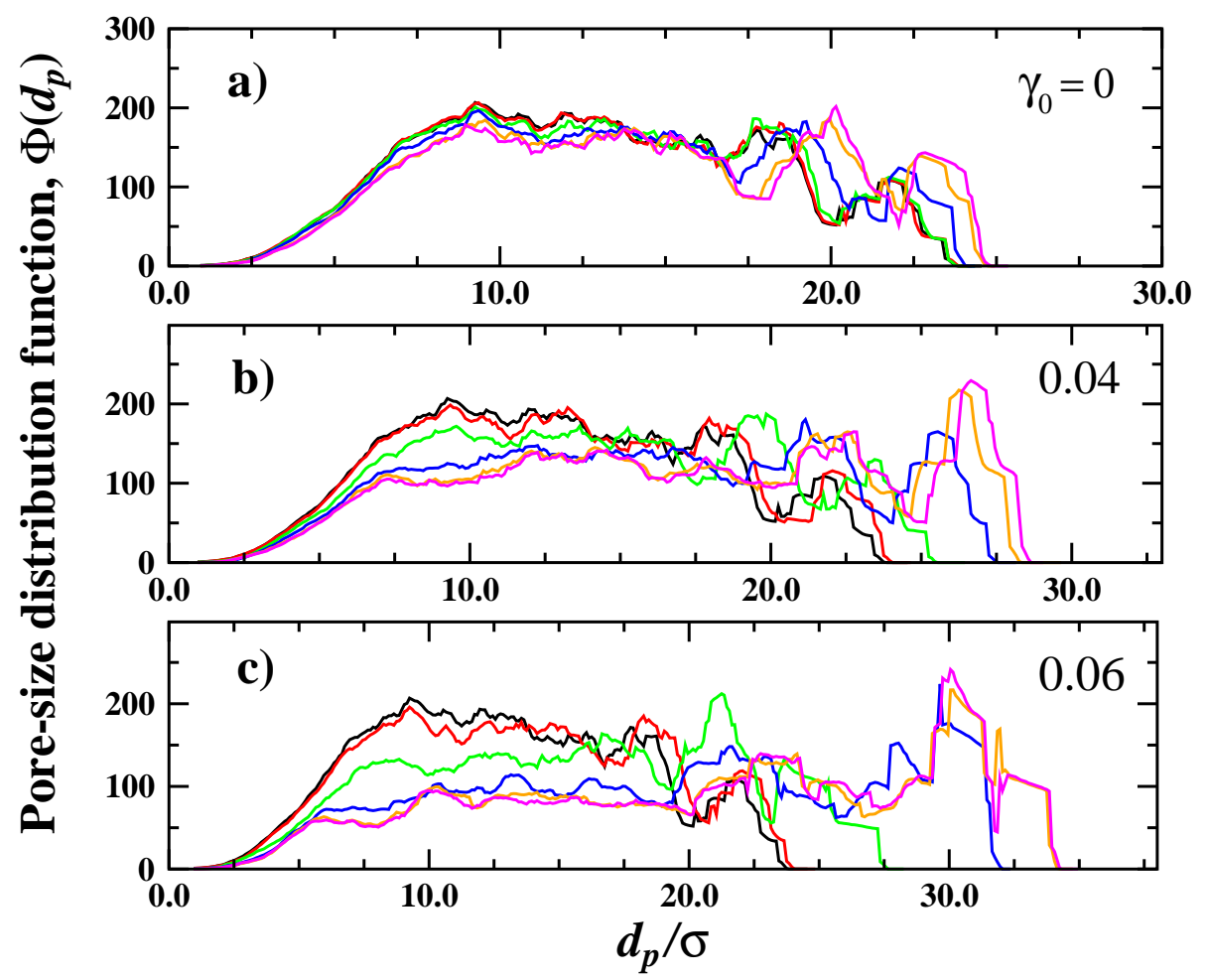

FIG. 8: (Color online) The pore size distribution (PSD) functions for the strain amplitudes (a) $\gamma_{0}=0$, (b) $\gamma_{0}=0.04$, and (c) $\gamma_{0}=0.06$. The colorcode for the cycle number is as follows: 0 (black), 1 (red), 10 (green), 100 (blue), 1000 (orange), and 2000 (magenta). 


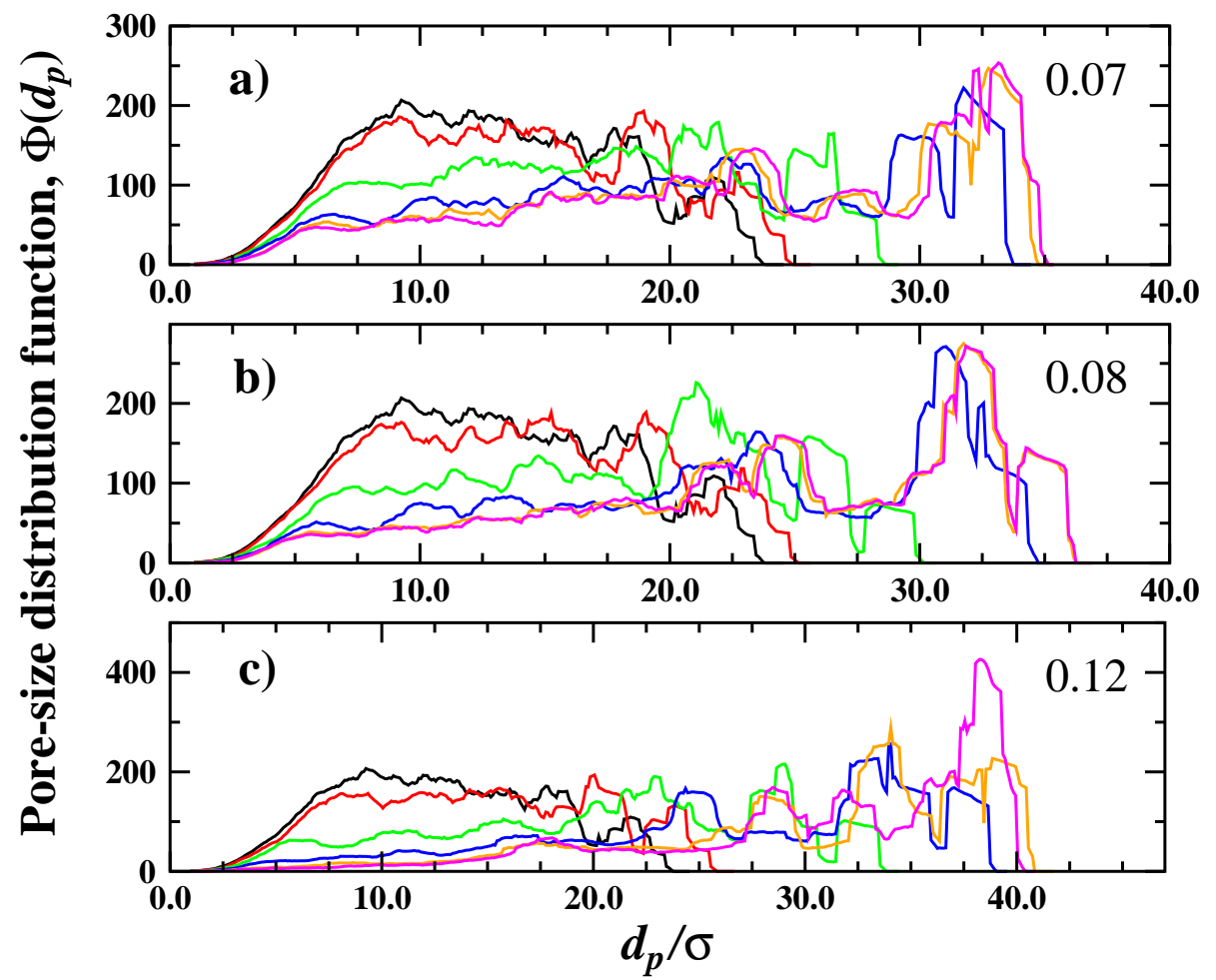

FIG. 9: (Color online) The pore size distribution functions for the strain amplitudes (a) $\gamma_{0}=0.07$, (b) $\gamma_{0}=0.08$, and (c) $\gamma_{0}=0.12$. The colorcode for the cycle number is 0 (black), 1 (red), 10 (green), 100 (blue), 1000 (orange), and 2000 (magenta). 

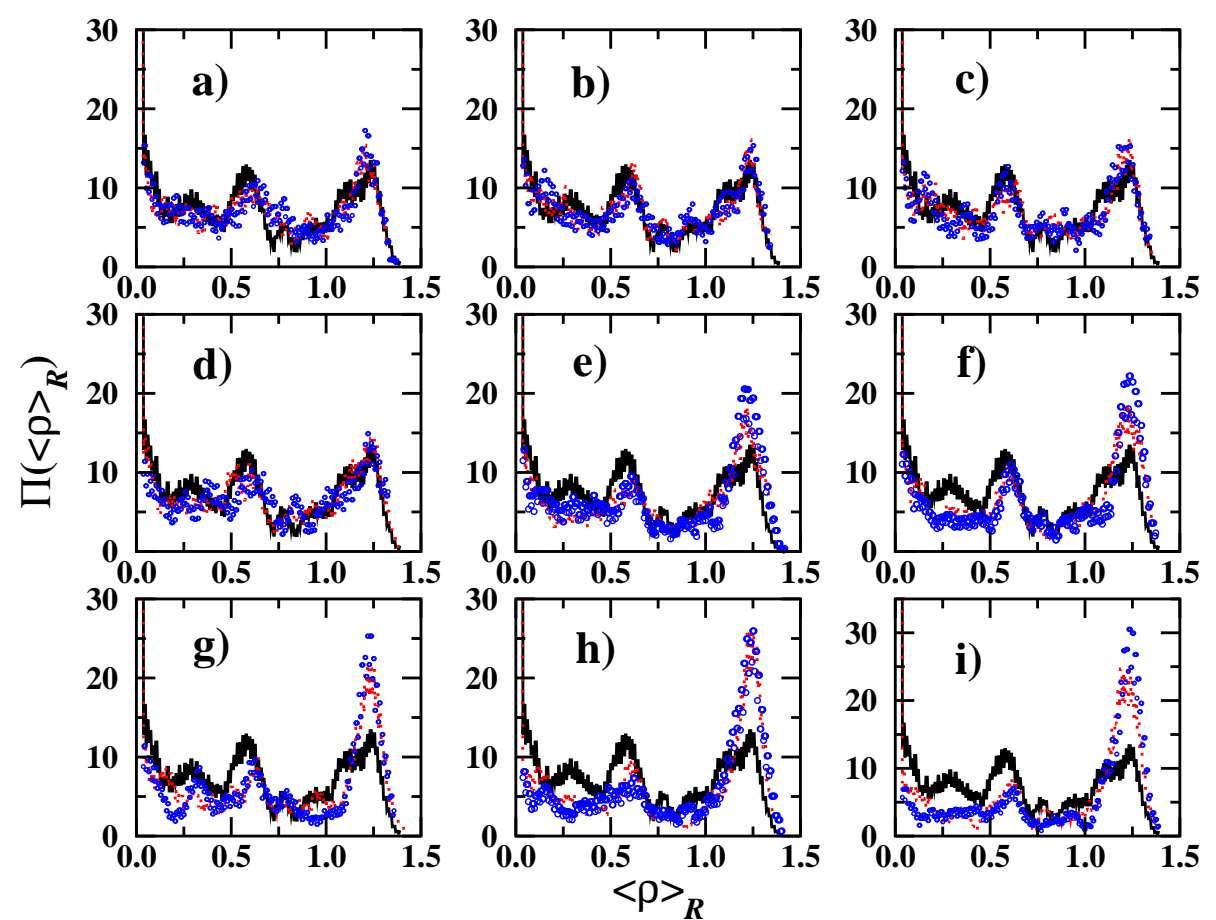

FIG. 10: (Color online) On-lattice local density distribution functions, $\Pi\left(\langle\rho\rangle_{R}\right)$, for the selected strain amplitudes, $\gamma_{0}$ : (a) 0.0, (b) 0.01, (c) 0.02, (d) 0.04, (e) 0.06, (f) 0.07, (g) 0.08, (h) 0.10, and (i) 0.12. The distributions were computed using the bin size $\langle\rho\rangle_{R}^{\max } / 400$ and each set of data was averaged over 20 data points for clarity. In each panel, we show the density distribution functions computed at loading cycle number: 0 (solid black line), $100 T$ (dashed red line), and $2000 T$ (open blue circles). 